synthesis. 18 papers were deemed to be 'high' quality, five 'medium', the rest 'low'. Meaningful statistical associations were observed between the exposure (childhood SEP) and the outcome (ACEs/maltreatment) in the vast majority of studies, including all bar one of those deemed to be high quality. Low SEP is therefore clearly a determinant of ACEs/maltreatment: the longitudinal nature of many studies means the association is most likely causal.

Conclusion The relationship between childhood SEP and ACEs is clear, but under-researched. More evidence exists in the maltreatment literature. With UK child poverty levels predicted to increase markedly, any policy approach which ignores the socio-economic context to ACEs is flawed. Policy needs to help those currently affected by childhood adversity; but to prevent further adversity, it must also address the key socioeconomic drivers.

\section{OP63 TRAJECTORIES OF SOCIO-ECONOMIC POSITION FROM BIRTH TO ADULT AGE AND SUBSEQUENT MORTALITY: THE UPPSALA BIRTH COHORT MULTIGENERATIONAL STUDY}

${ }^{1,2}$ | Koupil*, ${ }^{1} \mathrm{~A}$ Heshmati, ${ }^{1,3} \mathrm{~A}$ Goodman, ${ }^{4} \mathrm{G}$ Mishra. ${ }^{1}$ Dept. Public Health Sciences, Stockholm University, Stockholm, Sweden; ${ }^{2}$ Dept. Public Health Sciences, Karolinska Institutet, Stockholm, Sweden; ${ }^{3}$ Faculty of Epidemiology and Population Health, London School of Hygiene and Tropical Medicine, London, UK; ${ }^{4}$ School of Public Health, University of Queensland, Brisbane, Australia

\subsection{6/jech-2019-SSMabstracts.42}

Background Several theoretical life course models (critical period, sensitive period, accumulation) have been proposed, all of which may be relevant for understanding of when and how socioeconomic inequalities in health arise. Our aim was to investigate whether the effect of socio-economic position on all-cause mortality accumulates over the life course or if some periods of the life course are more important than others.

Methods We followed 3,951 men and 3,601 women born in Uppsala, Sweden, in 1915-1929 with known SEP at birth (age $0)$, during childhood (10 years), in adulthood (30-45 years) and in later life (50-65 years) from September 1980 until emigration, death, or until December 2010. Data on parents', partner's and own occupational status (a measure of SEP), marital status, deaths and emigrations were abstracted from birth records, parish records, school records, Census 1930 and routine registers. From the eligible sample who were alive and living in Sweden in September $1980 \quad(n=11,336), \quad 67 \%$ $(n=7552)$ had SEP recorded at all four-time points. We compared a set of nested Cox proportional regression models, each corresponding to a specific life course model (critical, sensitive and accumulation models), to a fully saturated model, to ascertain which model best describes the relationship between SEP and mortality. An alternative analysis employed latent class trajectories of SEP across same four time points. Analyses were stratified by gender.

Results The effect of SEP across the life course on all-cause mortality was best described by the sensitive period model in both genders with social advantage in later life (ages 50-65 years) having the largest protective effect (HR 0.80, 95\% CI $0.73-0.87$ in men and HR 0.82 , 95\% $0.75-0.91$ in women). A linear accumulation model also provided a good fit of the data for women. Only 5\% and 12\% of individuals experienced downward and upward social mobility during childhood respectively. The sensitive period model indicated that being advantaged at age 10 appeared to be more protective than at birth for males, while there was no difference between SEP at birth and age 10 in their effect on all-cause mortality among women. Additional adjustments for marital status did not affect the results appreciably and main results were also consistent with analyses that employed latent class trajectories of SEP.

Conclusion Our results lead to a conclusion that an individual's socio-economic position over the life course, including during early childhood does affect their risk of all-cause mortality in later life. These findings indicate that improvements in social conditions at any stage of the life course can contribute to reducing mortality at old age.

\section{OP41 THE HEALTH OF PEOPLE EXPERIENCING MULTIPLE FORMS OF SOCIAL EXCLUSION: A SYSTEMATIC REVIEW}

${ }^{1}$ EJ Tweed $*,{ }^{2} \mathrm{C}$ Sumpter, ${ }^{1} \mathrm{R}$ Thomson, ${ }^{3} \mathrm{D}$ Lewer, ${ }^{4} \mathrm{P}$ Southworth, ${ }^{5} \mathrm{~A}$ Kirolos, ${ }^{6} \mathrm{~A}$ Story, ${ }^{7} \mathrm{~A}$ Hayward, ${ }^{8} \mathrm{~S}$ Hwang, ${ }^{9} \mathrm{R}$ Aldridge, ${ }^{1} \mathrm{SV}$ Katikireddi. ${ }^{1} \mathrm{MRC} / \mathrm{CSO}$ Social and Public Health Sciences Unit, University of Glasgow, Glasgow, UK; ${ }^{2}$ Department of Public Health, NHS Forth Valley, Stirling, UK; ${ }^{3}$ Collaborative Centre for Inclusion Health, University College London, London, UK; ${ }^{4}$ NHS Dumfries and Galloway, UK; ${ }^{5}$ Usher Institute, University of Glasgow, Glasgow UK; ${ }^{6}$ University College London Collaborative Centre for Inclusion Health and UCLH Find and Treat Service London, UK; ${ }^{7}$ Institute of Epidemiology and Health Care, University College London, London, UK; ${ }^{8}$ Centre for Urban Health Solutions, St. Michael's Hospital and Department of Medicine, University of Toronto, Toronto, Canada; ${ }^{9}$ Institute of Health Informatics, University College London, London, UK

\subsection{6/jech-2019-SSMabstracts.43}

Background People with a history of homelessness, imprisonment, substance use, sex work, or serious mental illness experience much higher rates of ill-health and premature death than the general population. There is substantial overlap in these experiences in the population, and they may interact in important ways to influence health. However, the health outcomes associated with these experiences in combination have not previously been reviewed.

We therefore aimed to synthesise existing evidence on allcause mortality; cause-specific mortality; morbidity from conditions appearing in ICD-10; self-rated health; and quality of life among people with lifetime exposure to more than one of the following: homelessness; imprisonment; substance use; sex work; or serious mental illness.

Methods We searched Medline, Embase, and Psycinfo using search terms for the above exposures and outcomes, in consultation with a medical librarian. Eligible studies comprised peer-reviewed English-language articles from high-income countries published since 1998 reporting at least one relevant outcome for people with lifetime exposure to two or more exposures of interest, in comparison to people with one or no exposures. Screening was undertaken independently by two authors using Covidence, with risk of bias assessed using a modified Newcastle-Ottawa Scale. Findings were summarised using a pre-specified narrative synthesis plan. The protocol was registered with PROSPERO (CRD42018097189).

Results Searches retrieved 15,948 unique citations. After full text screening of 1,583 studies, initial results from 293 studies for which data extraction has been completed are presented here. Of these, $73 \%$ were cross-sectional studies. The most common exposure combinations were imprisonment \& substance use (33\%) and serious mental illness \& substance use (26\%); only 11 data points (1\%) reported outcomes associated 
with $>2$ exposures. Infectious diseases were by far the most common outcomes studied (44\%): blood-borne viruses alone accounted for $33 \%$ of all data points. The next most common outcomes were external causes, injury, and poisoning (16\%) and mental disorders (14\%): together with infections, these categories accounted for $73 \%$ of all data points.

Conclusion Existing research on the health of people experiencing multiple forms of social exclusion is dominated by cross-sectional studies examining a relatively limited set of exposures and outcomes. The lack of data on more than two exposures in combination; self-rated health or quality of life; or non-communicable diseases (e.g. cardiovascular disease and cancer) suggests that research to date may not reflect the true burden of ill-health in these populations. Limitations include restriction to peer-reviewed studies and risk of publication bias.

\section{OP43 A CASE CONTROL STUDY OF SOCIAL DETERMINANTS OF TUBERCULOSIS RISK IN WHITE UK-BORN ADULTS IN ENGLAND}

'P Nguipdop Djomo*, 'LC Rodrigues, ${ }^{1} \mathrm{PG}$ Smith, ${ }^{2}$ A Abubakar, ${ }^{1} \mathrm{P}$ Mangtani. 'Infectious Disease Epidemiology, London School of Hygiene and Tropical Medicine, London, UK; ${ }^{2}$ Institute for Global Health, University College London, London, UK

\subsection{6/jech-2019-SSMabstracts.44}

Background Tuberculosis (TB) remains a public health problem in the UK-born population of England, including among young adults. Ecological studies indicate that deprivation is an important risk factor for $\mathrm{TB}$, but there are few recent individual-level studies in high income countries that have investigated the association between poverty-related social determinants of health inequality $(\mathrm{SDH})$ and $\mathrm{TB}$.

Our objective was to measure the association between individual socio-economic status and social determinants of health, and $\mathrm{TB}$, taking into account the clustering of social risk factors in individuals, and to estimate the potential population impact on TB rates.

Methods Secondary analyses of a nationwide case-control study conducted among UK-born White adults aged 23 to 38 years at diagnosis of their first TB episode, and randomly selected age and sex frequency-matched community controls. Data on some SDH (education, household overcrowding, tobacco smoking, alcohol use, drugs use, and history of homelessness and prison) were collected in face-to-face interviews. Statistical analyses, using logistic regression models, was informed by a theoretical causal framework (Directed Acyclic Graph) of plausible inter-relationships between the measured social factors.

Results Overall, 681 TB cases and 1183 controls were recruited. The risk of $\mathrm{TB}$ was about four times higher in subjects whose formal education was up to GCSE O-levels or less compared to those with at least a university degree $(\mathrm{OR}=3.94 ; 95 \% \mathrm{CI}: 2.74 ; 5.67)$, after controlling for other TB risk factors (age, sex, BCG vaccination and stays in Africa or Asia for ${ }^{3} 3$ months). After simultaneously adjusting for these risk factors and all measured social determinants, higher TB risk was also independently associated with tobacco smoking, use of drugs (especially injectable drugs - OR=5.67; 95\% CI: $2.68 ; 11.98$ ), history of homelessness and deprivation in the area of residence. Population Attributable Fraction (PAF) estimates suggested that tobacco use and class-A drug use were, respectively, responsible for $18 \%$ and $15 \%$ of $\mathrm{TB}$ cases in the target population.

Conclusion The results provide insight into some of the mechanisms through which deprivation increases the risk of $\mathrm{TB}$ in the general population in England and support the argument for improved approaches to TB control efforts, such as integrated health and social services in high-risk young adult populations.

\section{OP44 DOES ETHNIC-RACIAL IDENTITY MODIFY THE EFFECTS OF RACISM ON AUSTRALIAN ABORIGINAL CHILDREN SOCIO-EMOTIONAL WELLBEING?}

DM Macedo*, ${ }^{2}$ LG Smithers, ${ }^{3}$ R Roberts, ${ }^{2}$ DG Haag, 'LM Jamieson. 'S School of Dentistry, University of Adelaide, Adelaide, Australia; ${ }^{2}$ School of Public Health, University of Adelaide, Adelaide, Australia; ${ }^{3}$ School of Psychology, University of Adelaide, Adelaide, Australia

\subsection{6/jech-2019-SSMabstracts.45}

Background Racism impacts the health and wellbeing of racial minorities across the lifespan. Ethnic-racial identity (ERI) has been suggested as a protective factor against adversity on racial minorities' wellbeing, however, findings vary between ethnic-racial groups. Research in the Aboriginal Australian context is still limited. The present study tests ERI affirmation as an effect-modifier of the longitudinal association between racism and Aboriginal Australian children's socio-emotional wellbeing (SEWB). Effect-sizes are hypothesized to be smaller among children with higher ERI affirmation.

Methods Children $(n=408)$ from the K-Cohort of the Longitudinal Study of Indigenous Children (LSIC) were included in the analysis. Data were collected through questionnaire-guided interviews in two time points, when children were 7-10 years and 9-12 years. Children's racism experience, SEWB (assessed by the Strengths and Difficulties Questionnaire), and confounders were reported by caregivers. Information on ERI affirmation was provided through child self-report. Analyses were conducted in Stata 14. Multiple imputation with chained equations was used to address potential bias due to missing data. Poisson regression with robust errors estimated adjusted Risk Ratios (RRa) for the longitudinal effect of racism on different domains of child SEWB. For the effect-measure modification analysis, $\mathrm{RR}_{\mathrm{a}}$ were obtained for the different stratum of the exposure (racism) and the effect-modifier (ERI affirmation). The direction of the modification was indicated by the Relative Excess Risk due to Interaction (RERI) on the additive scale.

Results Children exposed to racism and with low ERI affirmation were at increased risk of increased socio-emotional difficulties, especially for hyperactive behaviour ( $\mathrm{RRa} 2.16$, 95\% CI 1.00, 4.67), conduct problems (RRa 1.76, 95\% CI 0.71 , 3.83), and total difficulties ( $R R a 1.94,95 \%$ CI $0.92,4.11$ ), although the $95 \%$ confidence intervals were wide. Positive effect-measure modification was found in these domains, with respective RERIs of $1.08,1.39$, and 1.14 . Contrary to our hypothesis, children with high ERI affirmation were at increased risk of peer problems (RRa 1.80, 95\% CI 0.83 , 3.90). A negative effect-measure modification was found in this domain (RERI: -0.75).

Conclusion Results indicate that promoting ERI affirmation among Aboriginal Australian children might assist to mitigate the effects of racism on specific domains of child SEWB. 